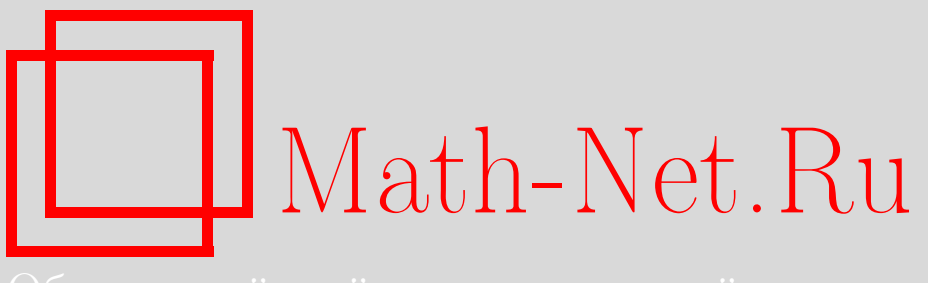

Общероссийский математический портал

Е. А. Печерский, Ю. М. Сухов, Идеи Р. Л. Добрушина в теории сетей обслуживания, УМН, 1997, том 52, выпуск 2, 25-30

DOI: https://doi.org/10.4213/rm1585 
Использование Общероссийского математического портала Math-Net.Ru подразумевает, что вы прочитали и согласны с пользовательским соглашением http://www.mathnet.ru/rus/agreement

Параметры загрузки:

IP: 54.198 .67 .100

26 апреля 2023 г., 14:48:03

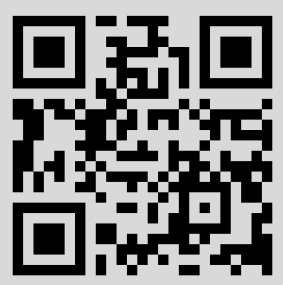




\section{ИДЕИ Р. Л. ДОБРУШИНА В ТЕОРИИ СЕТЕЙ ОБСЛУЖИВАНИЯ}

\section{Е. А. ПЕчеРСКИЙ, Ю. М. Сухов}

Насколько нам известно, Роланд Львович Добрушин начал активно интересоваться теорией сетей обслуживания с 1971 года. С чисто математической точки зрения эта теория служила для него естественной областью применения многочисленных идей, которые он успешно разрабатьвал в тот период (и позднее) в связи с задачами равновесной и неравновесной статистической механики и теории марковских процессов с локальным взаимодействием. Позднее обе теории он объединил под обшим названием теории многокомпонентных случайных систем. В частности, важную формирующую 
роль в добрушинском подходе к теории сетей сыграли его работы [3]-[5], а впоследствии [6]. Следует сказать, что его влияние на становление теории сетей обслуживания много шире, чем это можно было бы проследить по его публикациям в этой области. При этом влияние его идей и подходов испытьвали на себе не только сотрудники его лаборатории и его ученики.

Роланд Львович с самого начала ясно понимал важность многочисленных приложений возникающей в те годы теории, основанной на строгих результатах в статистической физике. Стоит отметить, что в течение 70-х годов основная масса специалистов в теории очередей продолжала работать в традиционном направлении, исследуя очереди к изолированному обслуживающему устройству. Существовало довольно распространенное мнение, что сети с очередями представляют слишком громоздкий обьект для строго математического исследования и создание здесь содержательной теории есть задача скорее завтрашнего дня. Действительно, до середины 70-х годов в теории сетей была известна единственная нетривиальная модель, поддающаяся до определенной степени строгому изучению, - модель Джексона [15], [16]. Дальнейшее продвижение было также связано с рядом ограничений на модели, которое затрудняло их применение (см. [14], [17], [18], а также обзор [13]).

Мы дадим очень короткое описание сети, изученной Джексоном, и его результатов. Сеть обслуживания проше всего мыслить как некоторый граф с ориентированными ребрами. Имеются случайные потоки требований (клиентов), приходящие на некоторые из узлов графа. Требование, пришедшее в некоторьй узел, имеет заданньй маршрут по вершинам графа, начинаюшийся в узле, в который требование пришло. Маршрут согласован с ориентацией ребер, соединяюших вершины маршрута. Требования обслуживаются в узлах своих маршрутов по тем или иным алгоритмам. Время обслуживания в каждом посешаемом узле есть случайная величина, зависяшая в обшем случае от маршрута. Джексон изучал сеть с независимыми пуассоновскими входньми потоками и экспоненциально распределенными временами обслуживания, которые зависят только от узла. Алгоритм обслуживания в каждом узле задавался естественным правилом "первьм пришел - первым обслуживаешся" (FCFS). Маршруты в модели Джексона задаются с помошюю субстохастической матрицы $\left(p_{i j}\right)$, где $p_{i j}$ - это вероятность перехода из узла с номером $i$ в узел с номером $j$. Если обозначить интенсивность входного потока в узел $j$ символом $\lambda_{j}$, то можно написать систему уравнений для интенсивностей $\rho_{j}$ потоков, поступаюших на каждый узел $j$, в которых кроме входного потока извне учтены также потоки в узел $j$ из узлов, связанных с узлом $j$ :

$$
\rho_{j}=\lambda_{j}+\sum_{1 \leqslant i \leqslant K} \rho_{i} p_{i j}, \quad j=1, \ldots, K
$$

где $K$ - число узлов в сети. По аналогии с классическими системами массового обслуживания, состояшими из одного прибора, естественно предположить, что сеть неперегруженна, если

$$
\rho_{j}<\mu_{j}, \quad j=1, \ldots, K,
$$


где $\mu_{j}$ - интенсивность обслуживания на узле $j$. Джексон доказал этот и серию других фактов о сетях, носяших его имя. Наиболее удивительньм оказался тот факт, что в стационарном режиме сеть Джексона работает (с точки зрения длин очередей в узлах), как система $K$ изолированных одноканальных систем с пуассоновским входньм потоком с интенсивностями $\rho_{j}$ и экспоненциально распределенными временами обслуживания со средним $\mu_{j}^{-1}$. Это тем более поразительно, что “полные” потоки клиентов, поступаюших на серверы, вообше говоря, зависимы и отнюдь не являются пуассоновскими (распределение потоков в сети Джексона по сей день неизвестно).

Нетрудно понять ограничительность этих моделей. Добрушин рассматривал их как модели аналогичные точно-решаемым моделям статистической физики. Поэтому он, хотя и предлагал, ослабляя условия на сеть, исследовать область, где еще действуют законы, открытые Джексоном, считал, что основные усилия здесь следует направить на построение качественной теории сетей (см. [2], [6], [8]), в которой законы Джексона могли бы появиться уже только в асимптотическом виде при том или ином предельном переходе. Понимая теорию сетей обслуживания как один из обьектов теории многокомпонентных случайных систем, он предлагал использовать здесь термодинамический и гидродинамический предельные переходы. Он также подчеркивал, что большая группа симметрий, обычно присущая сетям обслуживания, позволяет использовать развитьй в рамках статистической физики метод среднего поля. Мы остановимся здесь на нескольких результатах, полученных с использованием этих и других идей, активно эксплуатируемых в статистической физике.

В работе [8] Добрушин сделал первый шаг в использовании термодинамического предельного перехода для изучения проблемы устойчивости работы большой сети в стационарном режиме. Формально это сводится к исследованию единственности стационарного режима, что осмысленно только для бесконечных сетей. В этой работе исследовалась одномерная бесконечная сеть и было показано, что при малой интенсивности потока сообщений стационарный режим единственньй. Развитие эта деятельность нашла в работе [20], где исследовалась бесконечная сеть обслуживания с неединственным стационарным режимом работы.

Добрушин настойчиво пропагандировал также использование гидродинамического предельного перехода при исследовании сетей обслуживания. Не существует его работ на эту тему, однако эта область активно развивается. По-видимому первый шаг был сделан в работе [21]. Это один из примеров его влияния, которое невозможно измерить опубликованными или оставшимися в рукописи его работами. О плодотворности этой идеи можно судить по количеству публикаций, использующих этот метод (см. [22]).

Его активная пропаганда метода среднего поля также дала плоды, которые нельзя измерить только публикациями. Но в этой области имеются опубликованные его работы [6], [2]. Мы остановимся на последней работе, в которой Роланд Львович принял участие уже в последние месящы жизни.

Рассмотрим систему с $K$ серверами $S_{1}, \ldots, S_{K}$, в которую поступает пуассоновский поток интенсивности $\lambda K, \lambda<1$. В момент поступления клиент производит наудачу выбор (с возврашением) двух серверов среди $S_{1}, \ldots, S_{K}$, а затем поступает на обслуживание в тот из двух отобранных, где длина очереди менше. Длительности 
обслуживания предполагаются независимыми и экспоненщиально распределенными, со средним 1 . Если $n_{j}$ - это число клиентов, стояших в очереди к устройству $j$, то в стационарном режиме

$$
\lim _{K \rightarrow \infty} \operatorname{Pr}\left(n_{j}>m\right)=(\lambda)^{2^{m}}
$$

Для сравнения рассмотрим случай, когда клиент просто выбирает наудачу один из серверов $S_{1}, \ldots, S_{K}$ (это эквивалентно ситуации, где имеется один изолированньй сервер, на которьй поступает поток интенсивности $\lambda$ ). В этом случае

$$
\lim _{K \rightarrow \infty} \operatorname{Pr}\left(n_{j}>m\right)=\lambda^{m}, \quad m \geqslant 1 .
$$

Это означает, что даже сравнительно “либеральное” управление в сети позволяет резко уменшшить вероятность длинных очередей.

С конща 80-х Добрушин начинает интересоваться теорией больших уклонений. Он предлагает совершенно новьй взгляд на исследования больших флуктуаций в сетях обслуживания. Развиваемьй к этому времени подход приводил к исследованию принципа больших уклонений для кусочно однородных блужданий в конечномерных пространствах или их частях. Возникаюшие здесь трудности не преодолены в полной мере до сих пор. Хотя Роланд Львович опубликовал одну работу в этом направлении [1], он также предложил совершенно новый подход в этих проблемах. Его идея состояла в том, чтобы выразить изучаемьй функционал в терминах входного потока. Тогда событие, состояшее в том, что исследуемый функционал примет большое значение, также выражается в терминах входного потока. Это выражение может быть довольно сложным и очень редко имеет явную форму, однако такое сведение приводит к принципу больших уклонений для входного потока, что очень часто много проще упомянутого вьше принщипа больших уклонений для кусочно постоянных блужданий. Этим методом в работе [12] была изучена простейшая сеть, а именно, тандем. Мы приведем здесь этот результат. Входной поток в систему типа тандем может быть описан как последовательность независмых случайных векторов $\left(\xi_{n}^{1}, \xi_{n}^{2}, \tau_{n}\right)$. Здесь $\tau_{n}$ - это интервал времени между приходами $(n-1)$-го и $n$-го клиентов. В [12] величины $\tau_{n}$ распределены экспоненциально с параметром $\lambda$. С лучайные величины $\xi_{n}^{1}, \xi_{n}^{2}$ определяют времена обслуживания для $n$-го клиента на первом и втором приборах, соответственно. В paботе предполагается, что все три компоненты каждого вектора независимы. Главное условие на случайные величины $\xi_{n}^{1}$ и $\xi_{n}^{2}$ состоит в следующем. Существуют положительные $\theta_{+}^{1}$ и $\theta_{+}^{2}$ такие, что для любых $\theta<\theta_{+}^{i}$ конечно преобразование Лапласа:

$$
\varphi_{i}(\theta)=\mathrm{E} e^{\theta \xi_{1}^{i}}<\infty, \quad i=1,2 .
$$

Предполагается также, что система не перегружена, т.е. существует стационарньй режим ее работы. Для этого требуется, чтобы вьполнялись следующие два неравенства:

$$
\lambda \varphi_{i}^{\prime}(0)<1, \quad i=1,2
$$


В работе изучается функционал $\omega$, назьваемьй виртуальным временем ожидания. Это случайная величина, равная времени ожидания клиента, если бы он пришел в фиксированный момент времени. Так как система рассматривается в стационарном режиме, то распределение этой величины не зависит от выбранного момента времени. Результат работы следуюший. Для любого $a>0$

$$
\lim _{n \rightarrow \infty} \frac{1}{n} \ln \operatorname{Pr}(\omega>\text { an })=-\min \left\{\beta_{1}, \beta_{2}\right\}
$$

где $\beta_{1}$ и $\beta_{2}$ являются корнями уравнений

$$
\theta=\lambda\left[\varphi_{i}(\theta)-1\right], \quad i=1,2 .
$$

В этом результате виден также эффект, которьй на инженерном язьке назьвается "узкое место". Большие уклонения суммарного времени ожидания в тандеме сопадают с большими уклонениями на “худшем” обслуживаюшем устройстве, как если бы он работал независимо без другого устройства.

\section{СПИСОК ЛИТЕРАТУРЫ}

[1] Blinovsky V.M., Dobrushin R. L. Process level large deviations for a class piece-wise homogeneous random walks // The Dynkin Festshrift. Markov Processes and Their Applications / ed. M.I. Freidlin. Ser. Progress in Probability. V. 50. Boston: Birkhauser, 1994. P. 1-60.

[2] Введенская Н.Д. Добрушин Р. Л., Карпелевич Ф.И. Система обслуживания с выбором наименьшей из двух очередей - асимптотический подход // Пробл. передачи информ. 1996. Т. 32. № 1. С. 20-36.

[3] Добрушин Р. Л. О законе Пуассона для распределения частиц в пространстве // Укр. матем. журн. 1956. Т. 8. № 2. С. 127-134.

[4] Добрушин Р. Л. Марковские процессы с болшшим числом локально взаимодействующих компонент: существование предельного процесса и его эргодичность // Пробл. передачи инфоорм. 1971. Т. 7. № 2. С. 70-87.

[5] Добрушин Р. Л. Марковские процессы с большим числом локально взаимодействующих компонент: обратимьй случай и некоторые обобщения // Пробл. передачи информ. 1971. T. 7. № 3. C. 57-66.

[6] Добрушин Р. Л., Сухов Ю.М.Асимптотическое исследование звездообразных сетей коммутации сообщений с большим числом радиальных лучей // Пробл. передачи информ. 1976. Т. 12. № 1. С. $70-94$.

[7] Добрушин Р. Л., Сухов Ю.М.Временная асимптотика для некоторых вырожденных моделей эволюции систем с бесконечным числом частиц // Итоги науки и техники. Совр. пробл. матем.. Т. 4. М.: ВИНИТИ, 1979. С. 147-254.

[8] Добрушин Р. Л., Прелов В.В.Асимптотический подход к изучению сетей коммутации сообщений с болшшим числом частиц // Пробл. передачи информ. 1979. Т. 15. № 1. C. $61-73$.

[9] Добрушин Р. Л. Уравнение Власова // Функц. анал. и его прилож. 1979. Т. 13. № 2. C. $29-49$.

[10] Dobrushin R. L. Switching Networks, Gibbsian fields - interconnections // Proc. 1st World Congress of the Bernoulli Society (Tashkent, 1986). Utrecht: VN Sci. Press, 1987. P. 377-393. 
[11] Dobrushin R. L., Kelbert M. Ya., Rybko A. N., Suhov Yu. M. Qualitativemethods of queueing networks theory // Stochastic Cellular Systems: Ergodicity, Memory, Morphogenesis / ed. R.L. Dobrushin, V.I. Kryukov, A. L. Toom. Manchester: Univ. Press, 1990. P. 183-224.

[12] Dobrushin R. L., Pechersky E. A. Large Deviations for Tandem Queuing Systems // J. Appl. Math. Stochastic Anal. 1994. V. 7. P. 301-330.

[13] Baccelli F., Foss S., Mairess J. Stationary ergodic Jackson networks: results and counter-examples // Stochastic Networks. Theory and Applications / ed. F.P. Kelly, S. Zachary, I. Ziedens. Oxford: Clarendon Press, 1987. P. 377-393.

[14] Baskett F., Chandy K. M., Muntz R.R., Palacios F. G. Open, closed and mixed networks of queues with different classes of customers // J. Assoc. Comput. Mach. 1975. V. 22. № 2. P. 248-260.

[15] Jackson J. R. Networks of waiting times // Oper. Res. 1957. V. 5. № 4. P. 518-527.

[16] Jackson J.R. Jobshop-like queueing systems // Management Sci. 1965. V. 10.№ 1. P. 131-142.

[17] Kelly F. P. Networks of queues // Adv. Appl. Probab. 1976. V. 8. № 2. P. 416-432.

[18] Kelly F. P. Reversibility and Stochastic Networks. New York: Wiley, 1979.

[19] Wolf R. N. Stochastic Modelling and Theory of Queues. New York: Prentice-Hall, 1989.

[20] Кельберт М.Я., Конщевич М. А., Рыбко А. Н. О сетях Джексона на счетных графах // Теория вероятн. и ее примен. 1989. Т. 33. С. 358-361.

[21] Рыбко А.Н., Столяр А. Л. О эргодичности случайных процессов, описьвающих открытую сеть обслуживания // Пробл. передачи информ. 1992. Т. 28. С. 199-200.

[22] Kelly F. P., Williams R. J. (ed. ) Stochastic Networks. New York: Springer-Verlag, 1995. 